\title{
Effect of Anionic Polymers on Drug Loading and Release from Clindamycin Phosphate Solid Lipid Nanoparticles
}

\author{
Mohammadreza Abbaspour $^{1 *}$, Behzad Sharif Makhmalzadeh ${ }^{1,2}$, Zahra Arastoo $^{1}$ \\ Alireza Jahangiri ${ }^{1,2}$ and Roohollah Shiralipour ${ }^{2,3}$ \\ ${ }^{1}$ Nanotechnology Research Center and School of Pharmacy, ${ }^{2}$ Food and Drug Safety Evaluation Research Center, Ahvaz \\ Jundishapur University of Medical Sciences, ${ }^{3}$ Department of Chemistry, Shahid Chamran University, Ahvaz, Iran.
}

*For correspondence: Email: abbaspourmr@ajums.ac.ir; Tel/Fax: 0098-611-373-8380

\begin{abstract}
Purpose: To develop and characterize solid lipid nanoparticle (SLN) systems containing dextran sulfate or sodium alginate as anionic polymers for the delivery of clindamycin phosphate as a model hydrophilic cationic drug.

Methods: A multi-level factorial design was used for the preparation and optimization of clindamycin SLNs. Polymers (dextran sulfate and sodium alginate), Tween 80, and Pluronic F68 were chosen as the independent variables. The SLNs were prepared using stearic acid as the lipid matrix by an emulsion congealing technique with cold homogenization. Particle size and drug loading were evaluated as the primary responses. The morphology and drug release rate of the selected formulations were also determined.

Results: The results revealed that incorporation of anionic polymers increased drug loading of the SLNs. Dextran sulfate had a greater effect on drug loading, increasing it from 1.32 to $18.19 \%$, compared to the $6.73 \%$ achieved using sodium alginate. Dextran sulfate also reduced drug release rate by half compared with sodium alginate, probably due to the higher charge density, lower molecular weight and lower branching density of the ionic polymer.

Conclusion: Incorporation of anionic polymers can increase the loading of clindamycin phosphate into SLNs. Drug release from SLNs is also dependent on the polymer type.
\end{abstract}

Keywords: Clindamycin, Solid lipid nanoparticles, Dextran sulfate, Sodium alginate, Anionic polymers, Drug release, Drug loading

Tropical Journal of Pharmaceutical Research is indexed by Science Citation Index (SciSearch), Scopus, International Pharmaceutical Abstract, Chemical Abstracts, Embase, Index Copernicus, EBSCO, African Index Medicus, JournalSeek, Journal Citation Reports/Science Edition, Directory of Open Access Journals (DOAJ), African Journal Online, Bioline International, Open-J-Gate and Pharmacy Abstracts

\section{INTRODUCTION}

Solid lipid nanoparticles (SLNs) consist of nanosized solid lipids dispersed in an aqueous medium. SLNs combine the advantages and avoid the disadvantages of other colloidal carrier systems such as liposomes and polymer nanoparticles, which are known as alternatives to SLNs [1-3]. SLN formulations are adhesive, and they could prolong the residence time of the dosage form at the absorption site, consequently increasing its bioavailability [4]. Controlled drug delivery, enhanced bioavailability of entrapped drugs [5], and/or improved tissue distribution, good tolerability, and drug targeting have been attributed to SLN formulations [6].

Potential disadvantages such as an insufficient loading capacity, high initial burst release kinetics, drug leakage during storage, and relatively high water content of the dispersions $(70-99.9 \%)$ have been observed $[7,8]$. 
SLNs are usually applicable for entrapping lipophilic drugs $[9,10]$. However, for loading highly water-soluble, ionic drugs such as clindamycin, SLN formulations face the challenge of low drug partitioning in the lipid phase [11]. To deliver hydrophilic drugs, anionic polymers have been used to pair with cationic charged drugs to develop a polymer-lipid hybrid nanoparticle system, a variation of SLNs [12].

Clindamycin is a semi-synthetic antibiotic with a wide spectrum of activity used in the treatment of certain types of bacterial infections, including those of the lungs, skin, blood, female reproductive organs and eye. In this study, we used ion-pairing method for the preparation of SLNs to decrease the hydrophilicity of clindamycin phosphate as a model hydrophilic cationic drug via the application of anionic polymers (dextran sulfate or sodium alginate). The effect of type and amount of polymers, amount of drug and surfactants on particle size, drug loading and drug release from SLNs were studied. Furthermore, the characteristics of different formulations with and without polymers were compared.

\section{EXPERIMENTAL}

\section{Materials}

Clindamycin phosphate was purchased from Behvazan Pharmaceutical Company, Iran. Sodium alginate and dextran sulfate were obtained from Applichem, Germany. Tween 80 and stearic acid were purchased from Merck, Germany. Pluronic F68 was obtained from BASF, Germany. Acetonitrile was supplied by ROMIL-sps, UK. All chemicals were of analytical grade.

\section{Experimental design}

SLNs were prepared using a multi-level factorial design. Three independent variables including the amount of polymer (three levels), the amount of drug (two levels), and the amount of surfactants (two levels) were used (Table 1). The same amount of stearic acid was used in all formulations. The dependent variable or response was the drug loading. SPSS 16 software was employed for the experimental design and regression analysis of the data to evaluate the effect of the variables on the responses.

\section{Preparation of clindamycin-loaded SLNs}

SLNs were prepared using an emulsion congealing technique with cold high-pressure homogenization. The oil and aqueous phases were prepared separately. The oil phase consisted of stearic acid (100 mg) and half of the total amount of the surfactant and co-surfactant used in the formulation (20 or $40 \mathrm{mg}$ Tween 80 and Pluronic F68, based on the experimental design), while the aqueous phase consisted of 1 $\mathrm{mL}$ double-distilled water, the drug (clindamycin phosphate, 2 or $4 \mathrm{mg}$ ), the other half of the surfactant and co-surfactant, and the anionic polymer (2 or $4 \mathrm{mg}$ dextran sulfate or sodium alginate). The two phases were heated separately to $75{ }^{\circ} \mathrm{C}$ (almost $5^{\circ} \mathrm{C}$ higher than the melting point of stearic acid) for $10 \mathrm{~min}$. The aqueous phase was added to the oil phase and mixed using a magnetic stirrer at the same temperature for $30 \mathrm{~min}$. Afterward, the mixture was further treated by sonication $(90 \mathrm{~W})$ for 15 $\min$ at $75^{\circ} \mathrm{C}$. The emulsion was poured into cold water $\left(2-4^{\circ} \mathrm{C}\right)$ to a final volume of $25 \mathrm{ml}$ [10], and then the suspension was passed through a homogenizer (Emulsiflex-c3, Avestin, Canada) for five cycles (30 s for each cycle) at 20,000 bars.

Table 1: Independent variables (factors and levels for factorial design)

\begin{tabular}{lccc}
\hline \multirow{2}{*}{ Independent variable } & \multicolumn{3}{c}{ Levels } \\
\cline { 2 - 4 } & -1 & 0 & 1 \\
\hline $\mathrm{X}_{1}$ : Amount of polymer $(\mathrm{mg})$ & 0 & 2 & 4 \\
$\mathrm{X}_{2}$ : Amount of drug $(\mathrm{mg})$ & 2 & - & 4 \\
$\mathrm{X}_{3}$ : Amount of surfactants $(\mathrm{mg})$ & 40 & - & 80 \\
\hline
\end{tabular}

\section{Particle size determination}

The mean particle size and polydispersity index (PDI) of the SLNs were measured by photon correlation spectroscopy (PCS) (Scatteroscope 1, Qudix, Seoul, Korea) using a helium-neon laser with a wavelength of $633 \mathrm{~nm}$. Photon correlations of the spectroscopic measurements were determined at a scattering of $90^{\circ}$. A 1:100 dilution of the formulation was made using double-distilled water before the measurement. All determinations were made in triplicate.

\section{Scanning electron microscopy (SEM) of SLNs}

The surface morphology and stability of SLNs were studied by SEM (VP-1455, LEO, Germany). A drop of the SLN sample was applied evenly on the double-adhesive tape mounted on aluminium stub and dried at room temperature [9]. The dried sample was then sputter-coated by a thin layer of platinum under argon atmosphere using a sputter-coater (Polaron-England), and then examined by SEM. 
Determination of clindamycin phosphate concentration

HPLC determination of the clindamycin concentration was performed using an E4320V2 HPLC system (Knauer, Germany) at $220 \mathrm{~nm}$. A Nucleodur ${ }^{\circledR}$ column $(5 \mu \mathrm{m}, 250 \mathrm{~mm} \times 4.6 \mathrm{~mm}$, Macherey-Nagel, Germany) was used. The mobile phase consisted of phosphate buffer $(\mathrm{pH} 7)$ : acetonitrile $(775: 225)$ at a flow rate of 1.5 $\mathrm{mL} / \mathrm{min}$. An injection volume of $20 \mu \mathrm{L}$ was used, and clindamycin could be detected at a retention time of $3.5 \mathrm{~min}$ and a wavelength of $220 \mathrm{~nm}$. The linear correlation between the peak area and the clindamycin concentration $\left(R^{2}=0.9899\right)$ was obtained within the concentration range of $0.01-$ $6 \mathrm{mg} / \mathrm{mL}$, with a limit of quantification of 0.008 $\mathrm{mg} / \mathrm{mL}$.

\section{Determination of drug loading}

Following the preparation, the aqueous dispersion was centrifuged at $10,000 \mathrm{rpm}$ for 30 $\mathrm{min}$. The amount of free drug in the supernatant was determined by HPLC at $220 \mathrm{~nm}$. The amount of loaded drug was calculated by subtracting the amount of free drug from the initial drug amount. The drug loading efficiency (DLE) was calculated as in Eq 1.

DLE $(\%)=\{(\mathrm{Wi}-\mathrm{Wf}) / \mathrm{Wi}\} 100$

where $\mathrm{Wi}$ is the initial amount drug added in the dispersion and Wf the amount of free drug in the supernatant.

\section{In vitro drug release studies}

The method employed for the release investigation was the modified paddle method (USP type II apparatus). Phosphate buffer solution $(30 \mathrm{ml}, \mathrm{pH}$ 7) was placed in a modified $50 \mathrm{~mL}$ vessel and equilibrated at $37 \pm 0.5{ }^{\circ} \mathrm{C}$. SLNs were separated by centrifugation at 10,000 rpm for $30 \mathrm{~min}$. the SLN sediments were diluted with $1 \mathrm{~mL}$ distilled water and transferred to dissolution vessel..The speed of the rotation of the paddle was set at $100 \mathrm{rpm}$. The dissolution medium $(0.5 \mathrm{~mL})$ was withdrawn at $0.5,1,2,3$, $4,8,12,24,48,72,96$, and $120 \mathrm{~h}$ and analyzed by HPLC as described previously. A graph of \% cumulative release against time $(\mathrm{h})$ was plotted.

\section{Data analysis}

The effects of independent variables on the experimental response (drug loading) were modeled using a second order polynomial equation with a backward, stepwise linear regression technique. Only significant terms $(p<$ 0.05 ) were chosen for the final model. ANOVA and modeling process were performed using SPSS for Windows ${ }^{\circledR}$, Version 16.0 (SPSS Inc., USA). The related surface plots were obtained by Statgraphics for Windows ${ }^{\circledR}$, Version 5.1 plus (Statistical Graphics Corp., USA).

\section{RESULTS}

\section{Particle size and morphology}

The particle size of different formulations and their PDI are presented in Table 2. Their particle size ranged from $400-900 \mathrm{~nm}$ for all formulations and no clear pattern was observed. Furthermore, PDI was $<0.5$ for all formulations. SEM photographs of clindamycin SLNs showed they were semispherical with a size range which was consistent with PCS measurements (data not shown).

Table 2: Particle size, polydispersity index (PDI) and drug loading of SLN formulations

\begin{tabular}{l|ccc|ccc}
\hline \multirow{2}{*}{$\begin{array}{l}\text { Formulation } \\
\text { no. }\end{array}$} & \multicolumn{3}{|c|}{ SLN-ALG } & \multicolumn{3}{c}{ SLN-DS } \\
\cline { 2 - 6 } & $\begin{array}{c}\text { Particle size } \\
(\mathbf{n m})\end{array}$ & $\boldsymbol{P D I}$ & $\begin{array}{c}\text { Drug loading } \\
(\%)\end{array}$ & $\begin{array}{c}\text { Particle size } \\
(\mathbf{n m})\end{array}$ & $\boldsymbol{P D I}$ & Drug loading (\%) \\
\hline 1 & $38 \pm 482$ & 0.34 & $1.32 \pm 4.07$ & $38 \pm 482$ & 0.34 & $1.32 \pm 4.07$ \\
2 & $18 \pm 570$ & 0.34 & $0.40 \pm 0.29$ & $18 \pm 570$ & 0.34 & $0.29 \pm 0.40$ \\
3 & $51 \pm 457$ & 0.36 & $0.35 \pm 0.13$ & $51 \pm 457$ & 0.36 & $0.35 \pm 0.13$ \\
4 & $28 \pm 705$ & 0.31 & $5.92 \pm 0.44$ & $34 \pm 705$ & 0.31 & $5.92 \pm 0.44$ \\
5 & $45 \pm 711$ & 0.33 & $2.99 \pm 1.00$ & $18 \pm 529$ & 0.31 & $8.28 \pm 1.85$ \\
6 & $25 \pm 714$ & 0.28 & $6.73 \pm 0.49$ & $34 \pm 324$ & 0.4 & $15.27 \pm 3.05$ \\
7 & $32 \pm 673$ & 0.36 & $3.23 \pm 0.79$ & $26 \pm 576$ & 0.38 & $14.12 \pm 0.15$ \\
8 & $38 \pm 501$ & 0.32 & $6.49 \pm 0.75$ & $53 \pm 582$ & 0.35 & $12.89 \pm 1.37$ \\
9 & $40 \pm 883$ & 0.29 & $2.61 \pm 0.89$ & $19 \pm 472$ & 0.28 & $6.76 \pm 1.50$ \\
10 & $33 \pm 893$ & 0.38 & $3.98 \pm 1.04$ & $28 \pm 275$ & 0.33 & $13.10 \pm 2.45$ \\
11 & $19 \pm 384$ & 0.41 & $4.15 \pm 0.66$ & $31 \pm 399$ & 0.33 & $15.95 \pm 0.76$ \\
12 & $21 \pm 439$ & 0.32 & $5.09 \pm 0.84$ & $50 \pm 603$ & 0.34 & $18.19 \pm 0.31$ \\
\hline
\end{tabular}



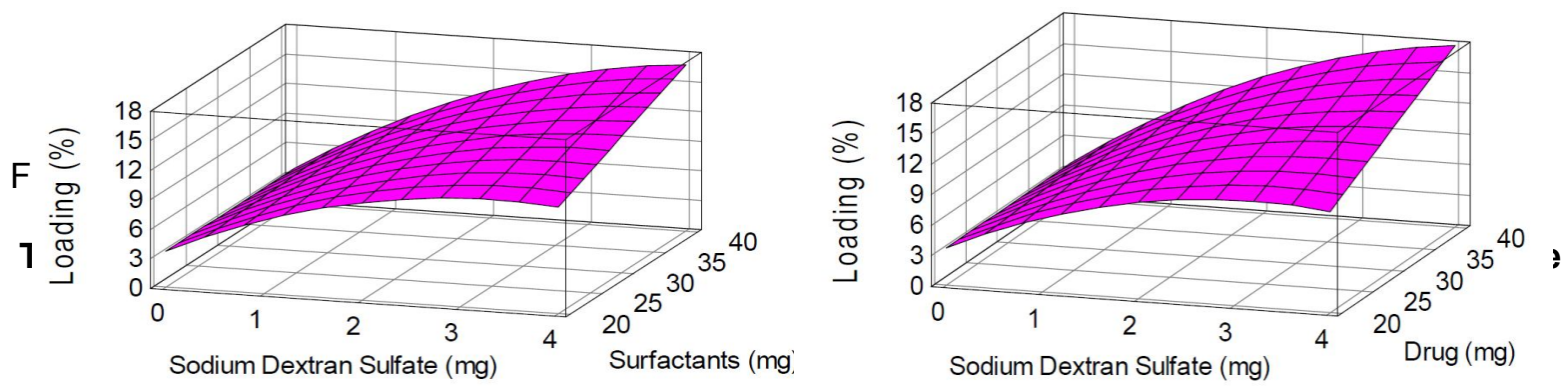

Fig 1: Effect of different factors on drug loading of dextran sulfate-containing SLNs

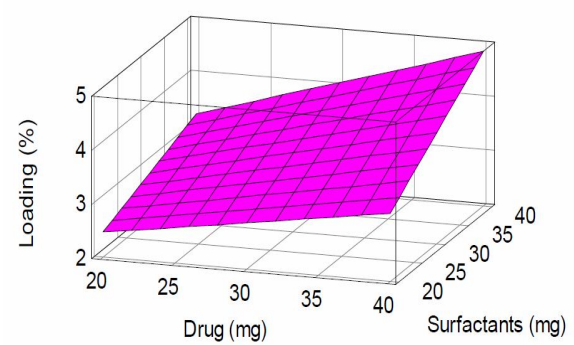

Fig. 2: Effect of the amounts of drug and surfactants on the drug loading of sodium alginate-containing SLNs

\section{Drug loading efficiency (DLE)}

Table 2 presents the drug loading of SLN formulations prepared using dextran sulfate and sodium alginate as the polymers and those prepared without a polymer. Analysis of the effect of variables and their interactions on response gave the mathematical models shown in Eqs 2 and 3.

$$
\begin{aligned}
D L E_{D S}= & 3.620-0.672 X_{1}^{2}+0.099 X_{1} X_{2}+0.076 X_{1} X_{3} \\
\mathrm{R}^{2}=0.921 &
\end{aligned}
$$

$D L E_{A L G}=1.656+0.002 X_{\mathbf{z}} X_{\mathbf{3}} \quad \mathrm{R}^{2}=0.528$

Clearly, three variables, viz, amounts of polymer, drug, and surfactant, were influential in the formulations containing dextran sulfate (Fig 1).

To maximize drug loading, all three variables must be included at their highest levels, and the results revealed that the amount of drug had a greater effect on loading than the amount of surfactants. Moreover, the loading of formulations containing sodium alginate was not affected by the amount of polymer, whereas the amounts of drug and surfactant affected the loading (Fig 2). The highest loading was obtained when the amounts of drug and surfactant were maximal. Formulations F12 containing dextran sulfate and F6 containing sodium alginate displayed a maximum loading of $18.19 \pm 0.31$ and $6.73 \pm 0.49 \%$, respectively. These two formulations were used for drug release studies.

\section{Drug release from SLNs}

The in vitro drug release profiles of the selected SLN formulations consisted of an initial burst release followed by a sustained and slow release (Fig 3).

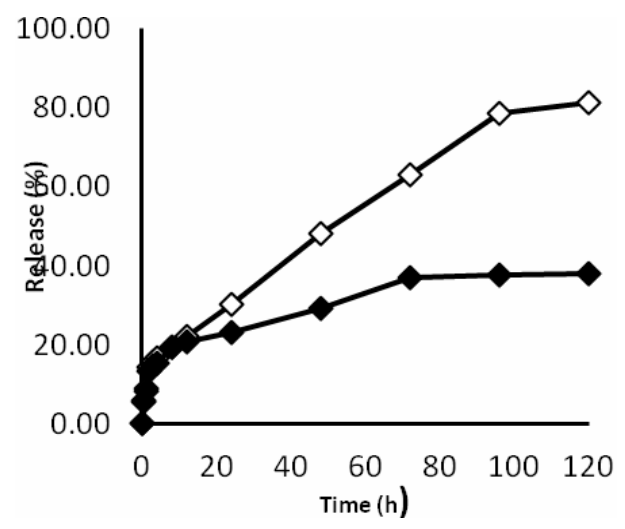

Fig 3: Profile of drug release from: $(\downarrow)$ formulation 12 (dextran sulfate) and $(\diamond)$ formulaton 6 (Sodium alginate)

For F12.DS, $13.46 \%$ of the drug was released in the first $2 \mathrm{~h}$, which was considered the burst release phase. By $120 \mathrm{~h}, 38.01 \pm 1.48 \%$ of the drug had been released. Conversely, F6.ALG displayed a burst release of $14.26 \%$ of the drug, and a maximum of $81.33 \pm 2.18 \%$ of the drug was released within $120 \mathrm{~h}$.

\section{DISCUSSION}

Particle size and distribution are considered important issues when formulating dispersed systems, particularly those intended for parenteral or ocular administration. The submicron-sized particles and solid state of physiological lipid carriers favor the pharmaceutical and biopharmaceutical applications of SLNs [8]. Nanoparticles have adhesive properties that could prolong the 
residence time of the drug in the administered area and increase its bioavailability.

In this study, particle size was not influenced by the polymer type or the amount of drug, polymer, or surfactant. However, in a previous study on the development of SLNs containing ionically complexed chemotherapeutic drugs, particle size appeared to be increased by the addition of the amount of polymer and drug [10]. This may be attributed to the additional cold-homogenization stage in the preparation method which can help to achieve partly uniform and narrow particle size distribution. The loading results were studied using a factorial design for the formulations separately prepared with dextran sulfate or sodium alginate. The results of the present study revealed that the presence of the polymer enhances the loading of hydrophilic drugs and that the type of polymer has a considerable effect on the amount of loading. In general, SLNs prepared with dextran sulfate as the polymer had a higher degree of loading than those made with sodium alginate. The ionic complex created between the polymers and clindamycin is necessary for the establishment of polymer hybrid SLNs. The complex between clindamycin and dextran sulfate or sodium alginate can be affected by the branching density, chain length, and degree of substitution [10]. It has been found that the encapsulation of cationic chemotherapeutics could be enhanced using dextran sulfate as an anionic polymer [10]. Dextran sulfate has a high degree of sulfate replacement, and the high density of dextran sulfate allows it to connect with cationic drugs, which results in higher drug loading in SLNs. In addition, the low molecular weight of dextran sulfate and the low density of its branches $(<5 \%)$ make it highly soluble in water, and thus, the complex between clindamycin and dextran sulfate is more complete [10].

The SLNs in the present investigation had a short period of burst release followed by a sustained and slow release, which is common in hydrophilic drug-containing SLNs [14,15]. In many cases, the burst release is due to the hydrophilic drugs accumulated at the o/w interface and in the outer shell during SLN preparation [16]. The prolonged release was due to the drug incorporated in the core. The ionic polymer can facilitate drug partitioning in the lipid phase, which can result in slower drug release. For example, increasing the amount of dextran sulfate in the formulations can reduce the initial burst release and slow the long-term release profile (Fig. 5).

\section{CONCLUSION}

Incorporation of anionic polymers can increase the loading of clindamycin phosphate into SLNs by decreasing the hydrophilicity of the drug. Dextran sulfate has significant effect on drug loading and a greater impact on prolonging drug release than sodium alginate.

\section{ACKNOWLEDGMENT}

This work is part of the Pharm.D. thesis of Ms. Z. Arastoo, which was supported by a grant from the Vice Chancellor of Research, Ahvaz Jundishapur University of Medical Sciences.

\section{REFERENCES}

1. Muller RH, Mader K, Gohla S. Solid lipid nanoparticles (SLN) for controlled drug delivery: a review of the state of art. Eur. J. Pharm. Biopharm. 2000; 50: 161-177.

2. Muller RH, Ruge SA. Solid lipid nanoparticles (SLN) for controlled drug delivery. In: Submicron emulsions in drug targeting and delivery. Harwood Academic Publishers, Amsterdam 1998: 219-234.

3. Gulati M, Grover M, Singh M. Lipophilic drug derivatives in liposomes. Int. J. Pharm. 1998; 165: 129-168.

4. Muller-Goymann CC. Physicochemical characterization of colloidal drug delivery systems such as reverse micelles, vesicles, liquid crystals and nanoparticles for topical administration. Eur. J. Pharm. Biopharm. 2004; 58: 343-356.

5. Demiral $M$, Yazan $Y$, Muller RH, Kilic $F$, Bozan $B$. Formulation and in vitro-in vivo evaluation of piribedil solid lipid nanoparticles. J. Microncapsul. 2001; 18: 359-371.

6. Coppert TM, Muller RH. Adsorption kinetics of plasma proteins on solid lipid nanoparticles for drug targeting. Int. J. Pharm. 2005; 302: 172-186.

7. Wissing SA, Kayser $\mathrm{O}$, Muller RH. Solid lipid nanoparticles for parenteral drug delivery. Adv. Drug Del. Rev. 2004; 56: 1257-1272.

8. Mehnert W, Mader K. Solid lipid nanoparticles, production, characterization and applications. Adv. Drug Del. Rev. 2001; 47: 165-197.

9. Sharif Makhmalzadeh B, Barati N, Hassani MH, Rahim F. Development of solid lipid nanoparticles as eschar delivery system for Nitrofurazone using taguchi design Approach. Int. J. Res. Pharm. Sci. 2010; 4: 466-472.

10. Wong HL, Bendayan $R$, Rauth AM, Wu XY. Development of solid lipid nanoparticles containing ionically complexed chemotherapeutic drugs and chemosensitizers. J. Pharm. Sci. 2004; 93: 19932008.

11. Ricketts CR. Dextran sulphate-a synthetic analogue of heparin. Biochem. J. 1952; 51: 129-133.

12. Mahner C, Lechner MD, Nordmeier E. Synthesis and characterisation of dextran and pullulan sulphate. Carbohydr. Res. 2001; 331: 203-208.

13. Li Y, Wong HL, Shuhendler AJ, Rauth AM, Wu XY. Molecular interactions, internal structure and drug release kinetics of rationally developed polymerlipid hybrid nanoparticles. J. Control. Release 2008; 128: 60-70.

14. Garcia-Fuentes, Torres D, Alonso MJ. New surfacemodified lipid nanoparticles as delivery vehicles for salmon calcitonin. Int. J. Pharm. 2005; 296: 122132. 
15. Hu FQ, Hong $Y$, Yuan $H$. Preparation and characterization of solid lipid nanoparticles containing peptide. Int. J. Pharm. 2004; 273: 29-35.
16. Almeida AJ, Souto E. Solid lipid nanoparticles as a drug delivery system for peptides and proteins. Adv. Drug Del. Rev. 2007; 59: 478-490. 